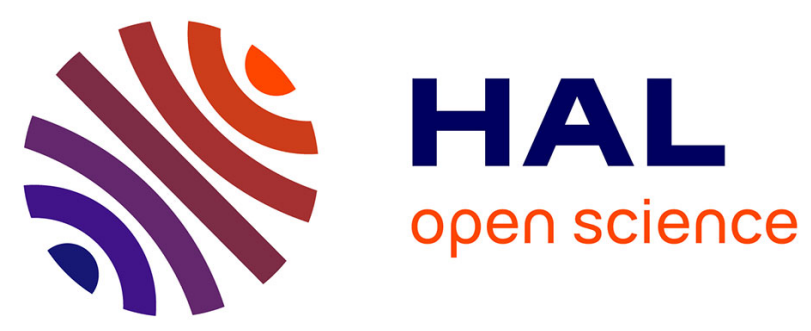

\title{
Acoustique d'un fluide au voisinage du point d'ébullition
}

Jean-Louis Auriault, Claude Boutin

\section{To cite this version:}

Jean-Louis Auriault, Claude Boutin. Acoustique d'un fluide au voisinage du point d'ébullition. Comptes Rendus de l'Académie des Sciences - Series IIB - Mechanics-Physics-Astronomy, 2000, 328 (2), pp.171-177. hal-00941058

\section{HAL Id: hal-00941058 https://hal.science/hal-00941058}

Submitted on 3 Feb 2014

HAL is a multi-disciplinary open access archive for the deposit and dissemination of scientific research documents, whether they are published or not. The documents may come from teaching and research institutions in France or abroad, or from public or private research centers.
L'archive ouverte pluridisciplinaire HAL, est destinée au dépôt et à la diffusion de documents scientifiques de niveau recherche, publiés ou non, émanant des établissements d'enseignement et de recherche français ou étrangers, des laboratoires publics ou privés. 


\title{
Acoustique d'un fluide au voisinage du point d'ébullition
}

\author{
Jean-Louis AURIAULT a , Claude BOUTIN ${ }^{\text {b }}$ \\ ${ }^{a}$ Laboratoire "Sols, Solides, Structures", UJF, INPG, CNRS UMR 5521, BP 53X, 38041 Grenoble cedex, \\ France \\ ${ }^{\mathrm{b}}$ Laboratoire géomatériaux, DGB URA CNRS 1652, ENTPE, rue Maurice-Audin, 69518 Vaulx en Velin, \\ France
}

Résumé. Dans une étude précédente (Boutin et Auriault [1]), la propagation d'ondes acoustiques dans un fluide à bulles en concentration finie était analysée au moyen de la méthode des échelles multiples. Trois comportements différents étaient mis en évidence suivant la taille des bulles, grande, moyenne ou petite. Dans la présente note, nous étendons cette étude par la prise en compte d'un possible changement de phase. Nous montrons que les effets de celui-ci sont négligeables dans le cas de grandes bulles, et modifient fortement le comportement des bulles moyennes en diminuant de plusieurs orders de grandeur la rigidité effective. Pour les petites bulles la capillarité devient le phénomène prépondérent.

fluide à bulles / acoustique / changement de phase

\section{Acoustics of bubbly liquids near the bubble point}

\begin{abstract}
In a previous paper ([1]) the homogenization technique of multiscale expansions was used for investigating how acoustic waves may propagate in a bubbly fuid at finite concentration. Three different equivalent macroscopic behaviours where derived, for "large", "medium" and "small" bubble systems, respectively. In the present paper, we extend the analysis by taking into consideration possible phase change effects. We show that phase change effects are negligible in the case of "large" bubbles, whereas they strongly modify the "medium" bubble system behaviour by decreasing the bulk modulus of several orders of magnitude. For "small" bubbles, capillary effects are dominating.
\end{abstract}

bubbly fluid / acoustics / phase change

\section{Abridged English version}

\section{Introduction}

The present note is an extension of [1]: the phenomena of phase change, which is of great importance in liquids near the bubble point, is added. The medium is assumed to be periodic, without lost of generality. The macroscopic equivalent models are determined by using the multiple scale expansion method [2]. In this method the bubble scale description is assumed to be given. The method is based on the existence of two well-separated characteristic lengths, $l$ and $L$, whose ratio is the small parameter used in the expansions. $l$ is of the order of the bubble size, whereas $L$ is related to the wavelength $\frac{l}{L}=\varepsilon \ll 1$. For the sake of simplicity, 
we assume that water is at atmospheric pressure and that the temperature is $T \approx 370^{\circ} \mathrm{K}$. Bubbles are vapor bubbles. The method of homogenization is described in [1]. The main result of this work is that phase change drastically changes the behaviour of medium-size bubbles.

\section{Physics at the bubble scale}

A very low perturbation of amplitude $\Phi_{\alpha}, \alpha=1, \mathrm{v}$, where 1 and $\mathrm{v}$ are for the liquid and the vapor, respectively, is superposed to an equilibrium state $\Phi_{\alpha}^{\mathrm{e}}$, see relation (1). Momentum, energy and mass balances, together with the equaiton of state of the vapor and the capillary equilibrium are given in [1]. The surface $\Gamma$ is assumed to be in thermodynamic equilibrium, and its temperature $T_{\Gamma}$ is given by the ClausiusClapeyron relation (2). Changes from [1] are given by heat flux and the displacement conditions (3) and (4) on $\Gamma$. The behaviour of the mixture is driven by Clausius-Clapeyron relation (2) which links the mechanical behaviour to heat transfer.

\section{Estimations}

The parameters are given in Table 1 . The bubble concentration is $\beta=O(1)$, the equilibrium pressure is $P^{\mathrm{e}} \approx 10^{5} \mathrm{~Pa}$, the entropy of vaporization is $L^{\varphi}=4.2 \cdot 10^{5} \mathrm{~J} \cdot \mathrm{kg}^{-1}$ and the capillary tension is $\sigma=$ $0.075 \mathrm{~N} \cdot \mathrm{m}^{-1}$. The dimensionless forms of the momentum, the energy and the mass balances, and of the equation of state of the vapor and the capillary equilibrium are given in [1]. They introduce three caracteristic bubbles sizes that are recalled in (5)-(7).

\subsection{Heat flux condition on $\Gamma$}

Introducing the heat layer thickness $l_{\alpha}^{\text {therm }}, \alpha=1, \mathrm{v}$, yields to estimation (8) for the flux ratio. ClausiusClapeyron relation gives $T_{\Gamma} / T_{\mathrm{v}}=O(1)$ and, from [1], we have $T_{1}=O\left(T_{\Gamma}\right)$. Therefore the flux ratio is estimated as in (9). The dimensionless form of the heat flux condition (3) takes the form (10).

\subsection{Bulk rigidity of the bubbly fluid}

An estimation (11) of the vaporization-condensation flux is obtained from (10). $H$ is a complex-valued heat memory function that depends on $\omega$ and that is due to the transient heat flow in the liquid. The corresponding amplitude $\theta_{\varphi}$ of the relative vapor volume produced by vaporization-condensation takes the form (12). The total relative vapor volume change $\theta_{\mathrm{v}}$ is the sum of the relative volume change $-p_{\mathrm{v}}^{0} / \gamma P_{\mathrm{v}}^{\mathrm{e}}$ in absence of phase change plus the relative volume change $\theta_{\varphi}$ due to phase change. Assuming an adiabatic process without loss of generality yields (13). When substituting $p_{\mathrm{v}}^{0}$ from (13) and the radius perturbation $r$ in (14) into the capillary equilibrium in (14) and denoting by $\theta=\beta \theta_{\mathrm{v}}$ the bulk deformation of the period, we obtain the bulk rigidity in the form (15). This form simplifies to relations (16)-(18) for large, medium and small bubbles, respectively. In the case of medium bubbles, the bulk rigidity is strongly decreased. In the case of small bubbles, the bulk rigidity becomes negative.

\subsection{Displacement condition on $\Gamma$}

From (10), we obtain (19). For large or medium bubbles, we have $p_{\mathrm{v}}^{0}=O\left(p_{1}^{0}\right)=O(\widetilde{K} \theta)$, and for small bubbles we obtain $p_{\mathrm{v}}^{0}=O\left(p_{\mathrm{c}}^{0}\right)$. Therefore, the dimensionless form of (4) is given by (20) for large and medium bubbles and by (21) for small bubbles, respectively.

\section{Macroscopic behaviour}

By following a similar analysis to [1], we obtain the macroscopic equations for describing the perturbation. Relations (22)-(24) are for large, medium and small bubbles, respectively. $M^{\prime}, M$ and $G$ (given in [1]) are functions of the pulsation. Phase change and heat transfer effects are negligible in (22), 
whereas they are important in (23). Capillary effects are dominant in (24). However, for very small bubbles, the Laplace relation for capillary equilibrium is questionable. Behaviour (23) is the richest one: (22) and (24) can be obtained from (23) in the limit.

\section{Introduction}

Nous étudions la perturbation acoustique d'un liquide près du point d'ébullition, contenant des bulles de vapeur de ce liquide à concentration finie. La présente note est une extension de l'analyse conduite dans [1], où seuls étaient pris en compte les effets mécanique, thermique et capillaire. Nous introduisons ici les effets du changement de phase. Le lecteur se reportera à [1] pour les détails concernant la méthode et le comportement local, à l'échelle des bulles. À cette échelle et sans entacher la généralité nous considérons le milieu comme périodique, de taille caractéristique $l$. L'onde acoustique introduit une deuxième longueur caractéristique $L$ liée à la célérité $c$, la pulsation $\omega$ et la longueur d'onde $\lambda$ par $L=\frac{c}{\omega}=\frac{\lambda}{2 \pi}$. Nous supposons ici une bonne séparation d'échelles $\frac{l}{L}=\varepsilon \ll 1$, et nous utilisons la méthode des développements asymptotiques pour obtenir la description macroscopique équivalente [2]. Pour la simplicité de la présentation, le liquide est de l'eau à pression atmosphérique et à température $T \approx 370^{\circ} \mathrm{K}$.

La méthode consiste à rendre adimensionnelle la description à l'échelle des hétérogénéités, à évaluer les nombres sans dimension en fonction du paramètre $\varepsilon$, puis à rechercher les grandeurs physiques sous la forme de développements asymptotiques en puissance de $\varepsilon$. Le comportement macroscopique équivalent découle des conditions de compatibilité sur les termes des développements. On montre ici que le changement de phase perturbe de façon très importante le comportement des bulles de taille «moyenne ».

\section{Physique à l'échelle des bulles}

Une perturbation acoustique de petite amplitude et de fréquence constante est superposée à un équilibre initial. Le paramètre de la perturbation est supposé assez petit pour ne pas interférer avec le processus d'homogénéisation. Toute quantité $\Phi$ peut être mise sous la forme :

$$
\Phi_{\alpha}^{\mathrm{t}}=\Phi_{\alpha}^{\mathrm{e}}+\Phi_{\alpha} \exp (\mathrm{i} \omega t), \quad\left|\Phi_{\alpha}\right| \ll\left|\Phi_{\alpha}^{\mathrm{e}}\right|
$$

où les exposants t et e signifient «total» et «équilibre»; $\alpha=1$ désigne le liquide et $\alpha=\mathrm{v}$ la vapeur. Les bilans des quantités d'accélération, d'énergie et de masse, l'équation d'état de la vapeur et l'équilibre capillaire sont donnés dans [1]. La surface $\Gamma$ des bulles est ici encore supposée être à l'équilibre thermodynamique, mais, en présence de changement de phase, sa température $T_{\Gamma}$ est donnée par la relation de Clausius-Clapeyron :

$$
T_{\mathrm{v}}=T_{1}=T_{\Gamma}=p_{\mathrm{v}} \frac{T^{\mathrm{e}}}{L^{\varphi} \rho_{\mathrm{v}}^{\mathrm{e}}}
$$

$L^{\varphi}$ est l'enthalpie de vaporisation. Le flux de chaleur est discontinu sur $\Gamma$ :

$$
k_{1} \operatorname{grad} T_{1} \cdot \boldsymbol{n}=k_{\mathrm{v}} \operatorname{grad} T_{\mathrm{v}} \cdot \boldsymbol{n}-L^{\varphi} \boldsymbol{j} \cdot \boldsymbol{n}
$$

$k$ est la conductivité, $\boldsymbol{n}$ est la normale unitaire extérieure de la bulle et $\boldsymbol{j}$ est le flux de masse de vaporisationcondensation. Enfin la dernière relation concerne les déplacements $\boldsymbol{u}$ sur $\Gamma$ :

$$
\mathrm{i} \omega \boldsymbol{u}_{\Gamma} \cdot \boldsymbol{n}=\mathrm{i} \omega \boldsymbol{u}_{1} \cdot \boldsymbol{n}-\frac{\boldsymbol{j} \cdot \boldsymbol{n}}{\rho_{\mathrm{l}}^{\mathrm{e}}}=\mathrm{i} \omega \boldsymbol{u}_{\mathrm{v}} \cdot \boldsymbol{n}-\frac{\boldsymbol{j} \cdot \boldsymbol{n}}{\rho_{\mathrm{v}}^{\mathrm{e}}}
$$

La physique est pilotée par la relation de Clausius-Clapeyron qui couple la mécanique à la thermique. 


\begin{tabular}{lcc}
\hline Milieu & Liquide & Vapeur \\
\hline Densité $\rho^{\mathrm{e}}\left(\mathrm{kg} \cdot \mathrm{m}^{-3}\right)$ & $10^{3}$ & 1,2 \\
Rigidité $\mathrm{K}(\mathrm{Pa})$ & $2 \cdot 10^{9}$ & $1,4 \cdot 10^{5}$ \\
Viscosité dynamique $\mu(\mathrm{Pa} \cdot \mathrm{s})$ & $10^{-3}$ & $20 \cdot 10^{-6}$ \\
Viscosité cinématique $\nu\left(\mathrm{m}^{2} \cdot \mathrm{s}^{-1}\right)$ & $10^{-6}$ & $15 \cdot 10^{-6}$ \\
Conductivité $k\left(\mathrm{~W} \cdot{ }^{\circ} \mathrm{K}^{-1} \cdot \mathrm{m}^{-1}\right)$ & 0,6 & 0,026 \\
Capacité de chaleur $C_{p}\left(\mathrm{~J} \cdot{ }^{\circ} \mathrm{K}^{-1} \cdot \mathrm{kg}^{-1}\right)$ & $4,18 \cdot 10^{3}$ & $10^{3}$ \\
Diffusivité $d\left(\mathrm{~m}^{2} \cdot \mathrm{s}^{-1}\right)$ & $1,4 \cdot 10^{-7}$ & $2,1 \cdot 10^{-5}$ \\
\hline
\end{tabular}

Tableau 1. Constantes caractéristiques du fluide à bulles.

Table 1. Caracteristic parameters of bubbly fluids.

\section{Estimations}

Nous considérons un fluide à bulles de rayon $R$, à concentration finie $\beta=O(1)$, à la pression atmosphérique $P^{\mathrm{e}} \approx 10^{5} \mathrm{~Pa}$, près du point d'ébullition $T^{\mathrm{e}} \approx 370^{\circ} \mathrm{K}$, supposé initialement au repos. Pour fixer les idées, nous utilisons les valeurs caractéristiques du tableau 1. L'enthalpie de vaporisation est $L^{\varphi}=4,2 \cdot 10^{5} \mathrm{~J} \cdot \mathrm{kg}^{-1}$. La tension capillaire est $\sigma=0,075 \mathrm{~N} \cdot \mathrm{m}^{-1}$. Les formes adimensionnelles des bilans de quantité d'accélération, d'énergie et de masse, de l'équation d'état de la vapeur et de l'équilibre capillaire sont celles données dans [1]. Ces formes dépendent de la taille des couches visqueuse $l^{\text {visc et }}$ thermique $l^{\text {therm }}$, avec $l^{\text {visc }}=O\left(l^{\text {therm }}\right)$ pour le liquide et/ou la vapeur. Trois cas sont mis en évidence :

- grandes bulles : les couches visqueuse et thermique sont petites par rapport à $R$. Le régime est adiabatique :

$$
l^{\mathrm{visc}}=O\left(l^{\mathrm{visc}}\right) \ll R
$$

- moyennes bulles : les couches visqueuse et thermique sont de l'ordre de $R$. Le régime thermique est transitoire :

$$
l^{\mathrm{visc}}=O\left(l^{\mathrm{visc}}\right)=O(R)
$$

- petites bulles : les couches visqueuse et thermique sont grandes par rapport à $R$. Le régime est isotherme :

$$
l^{\mathrm{visc}}=O\left(l^{\mathrm{visc}}\right) \gg R
$$

\subsection{Condition de flux thermique sur $\Gamma$}

En introduisant l'épaisseur de couche thermique $l_{\alpha}^{\text {therm }}=\sqrt{d_{\alpha} / w}, \alpha=1, \mathrm{v}$, le rapport des flux de chaleur sur $\Gamma$ peut être évalué par :

$$
\left(\frac{\left|k_{1} \operatorname{grad} T_{1}\right|}{\left|k_{\mathrm{v}} \operatorname{grad} T_{\mathrm{v}}\right|}\right)_{\Gamma}=O\left(\frac{k_{\mathrm{l}} l_{\mathrm{v}}^{\text {therm }}}{k_{\mathrm{v}} l_{1}^{\text {therm }}} \frac{\left|T_{1}-T_{\Gamma}\right|}{T_{\mathrm{v}}-T_{\Gamma}}\right)
$$

La relation de Clausius-Clapeyron donne $T_{\Gamma} / T_{\mathrm{v}}=T^{\mathrm{e}} C_{\mathrm{pv}} / L \varphi=O(1)$. D'autre part [1], la thermique du liquide est faiblement transitoire, $T_{1}=O\left(T_{\Gamma}\right)$. Finalement dans tous les cas, en accord avec Nakoriakov et al. [3] :

$$
\left(\frac{\left|k_{1} \operatorname{grad} T_{1}\right|}{\left|k_{\mathrm{v}} \operatorname{grad} T_{\mathrm{v}}\right|}\right)_{\Gamma}=O\left(\varepsilon^{-1}\right)
$$


On montre ainsi que le flux de chaleur est dominant dans le liquide. La conduction adimensionnelle de flux sur $\Gamma$ s'écrit :

$$
k_{1} \operatorname{grad} T_{1} \cdot \boldsymbol{n}=\varepsilon k_{\mathrm{v}} \operatorname{grad} T_{\mathrm{v}} \cdot \boldsymbol{n}-L^{\varphi} \boldsymbol{j} \cdot \boldsymbol{n}
$$

où nous avons conservé les notations des grandeurs physiques.

\subsection{Rigidité effective du fluide à bulles}

Évaluons tout d'abord le flux de vaporisation-condensation. Nous obtenons à partir de (10) et de l'équation de Fourier, et au premier ordre de grandeur noté par l'exposant 0

$$
4 \pi R^{2} \boldsymbol{j} \cdot \boldsymbol{n}=O\left(\mathcal{C} \omega \frac{p_{\mathrm{v}}^{0}}{P^{\mathrm{e}}} \Omega_{\mathrm{l}} \rho_{\mathrm{v}}^{\mathrm{e}}(1-H)\right), \quad \mathcal{C}=\frac{\rho_{\mathrm{l}}^{\mathrm{e}} C_{\mathrm{pl}} T^{\mathrm{e}} P^{\mathrm{e}}}{\left(\rho_{\mathrm{v}}^{\mathrm{e}} L^{\varphi}\right)^{2}} \approx 600
$$

$\mathcal{C}$ est un paramètre adimensionnel caractéristique de la mixture; $H$ est une mémoire thermique, fonction complexe de $\omega$, qui caractérise le flux thermique hors-équilibre dans le liquide; $1-H \ll 1$ (régime adiabatique), $1-H=O(1)$ et $H \ll 1$ (régime isotherme) respectivement pour les grandes, moyennes et petites bulles. L'amplitude $\theta_{\varphi}$ de la variation relative de volume de vapeur produite par vaporisationcondensation s'écrit :

$$
\theta_{\varphi}=-\boldsymbol{j} \cdot \boldsymbol{n} \frac{4 \pi R^{2}}{\mathrm{i} \omega \rho_{\mathrm{v}}^{\mathrm{e}} \Omega_{\mathrm{v}}}=O\left(-\frac{1-\beta}{\beta} \mathcal{C} \frac{p_{\mathrm{v}}^{0}}{P^{\mathrm{e}}}(1-H)\right)
$$

L'amplitude totale $\theta_{\mathrm{v}}$ de la variation relative de volume est la somme de la variation relative de volume en absence de changement de phase et de la variation relative due au changement de phase. En supposant un comportement adiabatique du gaz, sans nuire à la généralité, il vient :

$$
\theta_{\mathrm{v}}=O\left(-\frac{p_{\mathrm{v}}^{0}}{\gamma P_{\mathrm{v}}^{\mathrm{e}}}+\theta_{\varphi}\right)=O\left(-\frac{p_{\mathrm{v}}^{0}}{\gamma P^{\mathrm{e}}}\left(\frac{P^{\mathrm{e}}}{P_{\mathrm{v}}^{\mathrm{e}}}+\frac{1-\beta}{\beta} \mathcal{C} \gamma(1-H)\right)\right)
$$

La perturbation de la loi de Laplace s'écrit sur $\Gamma(c f .[1])$ :

$$
p_{\mathrm{l}}^{0}=p_{\mathrm{v}}^{0}+\frac{2 \sigma r}{R^{2}}, \quad r=\frac{R \theta_{\mathrm{v}}}{3}
$$

où $p_{\mathrm{v}}^{0}$ est lié à $\theta_{\mathrm{v}}$ par (13) et $r$ est la variation du rayon $R$ des bulles. Après substitution de $p_{\mathrm{v}}^{0}$ et $r$ dans (14) et notant $\theta=\beta \theta_{\mathrm{v}}$ la variation de volume de la période, nous obtenons (15) où $\widetilde{K}$ est la rigidité volumique :

$$
p_{1}^{0}=-\tilde{K} \theta, \quad \widetilde{K}=O\left(\frac{\gamma P^{\mathrm{e}}}{\beta\left(\frac{P^{\mathrm{e}}}{P^{\mathrm{e}}+2 \sigma / R}+\gamma \mathcal{C} \frac{1-\beta}{\beta}(1-H)\right)}-\frac{2 \sigma}{3 \beta R}\right)
$$

Trois comportements différents peuvent être distingués suivant les valeurs de $H$ et de $K_{c}=\frac{2 \sigma}{3 R}$, compte tenu de $\mathcal{C} \approx 600$ :

- grandes bulles : $K_{c}$ est négligeable ainsi que le changement de phase :

$$
\widetilde{K}=O\left(\frac{\gamma P^{\mathrm{e}}}{\beta}\right)=O\left(P^{\mathrm{e}}\right)
$$

- moyennes bulles : le changement de phase domine :

$$
\widetilde{K}=O\left(\frac{P^{\mathrm{e}}}{(1-\beta) \mathcal{C}(1-H)}\right) \ll P^{\mathrm{e}}
$$


Le changement de phase fait chuter la rigidité. Dans le cas présent l'ordre de grandeur de $\widetilde{K}$ est très fortement réduit;

- petites bulles : les effets capillaires sont prépondérants :

$$
\widetilde{K}=O\left(-\frac{2 \sigma}{3 \beta R}\right)<0
$$

La rigidité volumique est négative. La perturbation est instable.

\subsection{Condition de déplacement sur $\Gamma$}

Partant à nouveau de l'estimation (11), il vient :

$$
\frac{\boldsymbol{j} \cdot \boldsymbol{n}}{\rho_{\mathrm{l}}^{\mathrm{e}} \omega u_{\mathrm{l}}}=O\left(\mathcal{C} \frac{\rho_{\mathrm{v}}^{\mathrm{e}}}{\rho_{\mathrm{l}}^{\mathrm{e}}} \frac{R}{u_{\mathrm{l}}} \frac{p_{\mathrm{v}}^{0}}{P^{\mathrm{e}}}(1-H)\right)=O\left(\varepsilon^{-1} \frac{p_{\mathrm{v}}^{0}}{P^{\mathrm{e}}}(1-H)\right)
$$

Pour les grandes et moyennes bulles $p_{\mathrm{v}}^{0}=O\left(p_{\mathrm{l}}^{0}\right)=O(\widetilde{K} \theta)$, et pour les petites bulles $p_{\mathrm{v}}^{0}=O\left(p_{\mathrm{c}}^{0}\right)$. Ainsi la condition adimensionnelle de déplacement (4) s'écrit

- grandes ou moyennes bulles : la conduction thermique est importante :

$$
\mathrm{i} \omega \boldsymbol{u}_{\Gamma} \cdot \boldsymbol{n}=\mathrm{i} \omega \boldsymbol{u}_{\mathrm{l}} \cdot \boldsymbol{n}-\varepsilon^{2} \frac{\boldsymbol{j} \cdot \boldsymbol{n}}{\rho_{\mathrm{l}}^{\mathrm{e}}}=\mathrm{i} \omega \boldsymbol{u}_{\mathrm{v}} \cdot \boldsymbol{n}-\varepsilon \frac{\boldsymbol{j} \cdot \boldsymbol{n}}{\rho_{\mathrm{v}}^{\mathrm{e}}}
$$

- petites bulles : le flux de vaporisation-condensation domine :

$$
\mathrm{i} \omega \boldsymbol{u}_{\Gamma} \cdot \boldsymbol{n}=\mathrm{i} \omega \boldsymbol{u}_{1} \cdot \boldsymbol{n}-\varepsilon \frac{\boldsymbol{j} \cdot \boldsymbol{n}}{\rho_{\mathrm{l}}^{\mathrm{e}}}=\mathrm{i} \omega \boldsymbol{u}_{\mathrm{v}} \cdot \boldsymbol{n}-\frac{\boldsymbol{j} \cdot \boldsymbol{n}}{\rho_{\mathrm{v}}^{\mathrm{e}}}
$$

\section{Comportement macroscopique}

En utilisant les résultats introduits ci-dessus et l'analyse conduite dans [1], on obtient les équations décrivant le comportement macroscopique de la perturbation. Celui-ci dépend fortement de la taille des bulles. Trois types de comportements sont alors mis en évidence :

- grandes bulles :

$$
\left[1+\beta\left(1+M^{\prime}\right)\right] \Delta P=-\beta \rho_{\mathrm{l}}^{\mathrm{e}} \omega^{2} \frac{P}{\gamma P^{\mathrm{e}}}
$$

Les effets de conduction thermique et de changement de phase sont négligeables. $M^{\prime}$, définie dans [1], est une fonction réelle de $\omega$ qui exprime le couplage inertiel entre liquide et bulle ;

- moyennes bulles : le changement de phase et les effets thermiques sont présents :

$$
\begin{gathered}
{[1+\beta(1+M)] \Delta P=\rho_{\mathrm{l}}^{\mathrm{e}} \omega^{2}} \\
\left(\frac{\beta}{P^{\mathrm{e}}}\left[-1+G\left(1-\frac{1}{\gamma}\right)\right]+\frac{\beta}{L^{\varphi} \rho_{\mathrm{v}}^{\mathrm{e}}}[1-G]+(1-\beta) \frac{\rho_{\mathrm{l}}^{\mathrm{e}} C_{\mathrm{pl}} T^{\mathrm{e}}}{\rho_{\mathrm{v}}^{\mathrm{e} 2} L^{\varphi}}(1-H)\right) P
\end{gathered}
$$

$M$ (couplage visco-inertiel) et $G$ (non-équilibre thermique des bulles), définies dans [1], sont des fonctions complexes de $\omega$.

- petites bulles : les effets capillaires sont prépondérants :

$$
\frac{(1-\beta) K_{\mathrm{c}}}{\beta} \Delta P=\rho_{\mathrm{l}}^{\mathrm{e}} \omega^{2}
$$

La rigidité volumique est négative. La perturbation ne se propage pas. 
Remarquons que l'étude a été conduite en supposant valide la loi de Laplace, qui est sujette à caution pour les très petites bulles. Le comportement (23) pour les bulles moyennes est le plus riche, en ce sens que (22) et (24) s'obtiennent à partir de (23) par passage à la limite.

\section{Références bibliographiques}

[1] Boutin C., Auriault J.-L., Acoustics of a bubbly fluid at large bubble concentration, Eur. J. Mech. B/ Fluids 3 (1993) 367-399.

[2] Sanchez-Palencia É., Non-homogeneous Media and Vibration Theory, Lecture Notes in Physics 127, Springer, 1980.

[3] Nakoryakov V.E., Pokusaev B.G., Schreiber I.R., Wave Propagation in Gas-Liquid Media, CRC Press, Boca Raton, 1987. 